\title{
Advances in characterization and modeling of cementitious materials: transport and volume change in cementitious materials
}

\author{
Narayanan Neithalath ${ }^{1}$ Gaurav Sant $^{2}$
}

Published online: 19 July 2017

(C) Indian Institute of Technology Madras 2017

Cement-based materials are ubiquitous in the construction world, with massive quantities of concrete used to cater to the ever-increasing demands of transportation, housing, and energy infrastructure. Portland cement concrete has undergone dramatic changes over the past few decades, prompted primarily by the desire to conserve precious natural resources and to create longer lasting, sustainable concrete structures. Along with advances in chemistry of cement and concrete, novel methods to characterize and test these materials have revealed fundamental information about these systems that in turn led to better and efficient material design of cementitious systems. Rapid advances in computing methods and speeds have also contributed to novel ways of modeling the response of complex, multiscale, multi-phase systems such as cement-based materials that exhibit highly non-linear spatio-temporal responses. Accurate prediction of properties of interest, including mechanical and transport, greatly helps optimized material design, thereby contributing to resource conservation, waste utilization, and consequently contributing to greater sustainability in the built environment.

The thematic issue of this journal on advances in characterization and modeling of cementitious materials, thus intends to provide the readership with cutting-edge research results on some of the above-mentioned topics that are of great interest to the scientific community. The thematic issue is divided into two parts, each containing five invited scientific articles authored by eminent

Narayanan Neithalath

Narayanan.Neithalath@asu.edu

1 Arizona State University, Tempe, AZ, USA

2 University of California Los Angeles, Los Angeles, CA, USA researchers in the respective fields. The first one, which constitutes the June issue focuses on Transport and Volume Change, which are key determinants of durability of concrete structures. The second one, due in September will cover novel materials and test methods.

The first paper in this issue concerns the modeling of unsaturated moisture flow in concrete. A dual permeability model that can more accurately model moisture flow in damaged concrete is explored along with a classical isothermal model in an attempt to ensure improved service-life predictions. A Lattice-Boltzmann model for prediction of permeability of saturated cement pastes, including those of blended cement pastes is presented in the second paper. The accuracy of the developed LatticeBoltzmann algorithms are compared against analytical solutions for simple geometries. The influences of properties of cement and the filler materials, degree of hydration, and liquid-to-solid ratio on the saturated permeability are elucidated using a three-dimensional microstructural model. The third paper in this issues attempts to bring clarity on the significance and role of bulk pore liquid pressure and disjoining pressure on the desiccation shrinkage of cementitious materials. A thermodynamics-based mechanistic approach is utilized to derive expressions for both the bulk liquid pressure and the disjoining pressure. The paper suggests that the pore liquid pressure is the primary mechanism associated with the desiccation shrinkage of cementitious materials. The binding of chloride ions in the cementitious matrix during ionic transport through concrete and the associated modeling is the topic of the fourth paper. The authors develop an alternative approach to incorporate chloride binding/ release processes in the modeling of ingress of external chlorides in concrete through the use of thermodynamic 
modeling of chemical reactions. The predictive ability of the thermodynamic calculations are ascertained using experimental data. The final paper in this issue investigates the feasibility of imaging the movement of water into partially saturated concrete using an advanced electrical method, viz., electrical resistance tomography (ERT). It is demonstrated that ERT can assist in monitoring and visualizing water movement within concrete.
These papers provide the latest and unique information with respect to moisture transport in cement-based materials, and explores novel means of visualizing and modeling them. We believe that the state-of-the-art research reported in these papers will be of immense use to the scientific community and hope that this leads to continued research and scientific contributions to this important area in cement-based materials research. 